\title{
Constituent Boundary Parsing for Kxample-Based Machine Translation
}

\author{
Osamu rURUSE and Hitoshi IID A
}

ATR Interpreting Telecommunications Research Laboratories

\begin{abstract}
This paper proposes an elfective parsing method for example-based machine transhatom. In this method, an input string is parsed by the top-down application of linguistic patterns consisting of variables and constituent boundaries. A constituent boundary is expressed by either a functional word or a part-ol-speech bigram. When strucural ambiguity occurs, the most plausible structure is selected using the total values of distance calculations in the example-based framework. Transfer-Driven Machine Translation (TDMT') achieves efficient and robust translation within the example-based framework by adopting this parsing method. Using bidirectional translation belween Japanese and Fnglish, the effectiveness of this method in TDMT is also shown.
\end{abstract}

\section{Introduction}

Example-based frameworks ane increasingly being applied to machine translation, since they can provide efficient and robust processing (Nagao, 1984; Sato, 1991; Sumita, 1992; Furuse, 1992; Watanabe, 1992). However, in order to make the best use of the advantages of an example-based framework, it is essential to effectively integrate an example-based method and source language analysis. Unfortunately, when an examplebased method is combined with a soure language analysis method having complex grammar rules, putting a heavy load on translation, the advantages of the example-based framework may be rumed. To achieve efficient and robust processing by the example-based framework, a lot of studies have been made for the purpose of combining source language analysis with an example-based method, and of efficiently covering the analyzed source language structure by means of transfor knowledge (Grishinan, 1992; Jones, 1992; MeLean, 1992; Maruyama, 1992, 1993; Nirenburg 1993).

One way to reduce the load of source language analysis is to directly apply transfer knowledge to an input string, which simulaneously executes both structural parsing and transfer knowledge application through pattern-matching. Pattern-matching does not use grammatical symbols such as "Noun Phrase", but uses surface words and non-grammatical symbols. "lherefore, in pattern-matching, rule competition is reduced, and linguistic structure is expressed in a simpler manner than in grammar-based parsing. Thus, pattem-matehing achieves efficient parsing. It is also useful in treating spoken language, which somelimes deviates from conventional grammar, while grammar-based parsing has difliculy treating unrestricted spoken language.

This paper proposes a constituent boundary parsing method based on pattern-matching, and shows its effectiveness for spoken language translation within the example-based lamework. In our parsing method, an input string is applied linguistic patterns expressing some linguistic constituents and their boundaries, in a top-down lashion. When structural ambiguity oceurs, the most plausible structure is selected using the total values of distance calculations in the example-based franework. Since the description of a linguistic pattem is simple, it is easy to update by adding reedback.

A constituent boundary parsing method using mutual information is proposed in (Magerman 1990). This method accounts for the unestricted natural language and is efficient. However, it lends to be inaccurate, and difficule to add feedback to, since it completely depends on statistical information withoul resort to a linguistic viewpoint. On the contrary, in order to achieve accurate parsing and translation, our conslituent boundary parsing method implicitly incorporates grammatical information into patterns, egr. constituent boundary description by a part-ol-specel bigram, and classification of patterns according to linguistic levels such as simple sentence and noun pluase.

Transler-Driven Machine Translation (T'DM'l) (Furuse, 1992, 1994) uses the constituent boundary parsing method presented in this paper, as an altemative to grammar-based analysis, and makes the best use of the example-based franework. A bidirectional translation system between Japanese and English for dialogue sentences conceming international conference registrations has been implemented (Sobashima, 1994). Ixperiments with the system have shown our parsing method to be eflective.

Section 2 defines patterns expressed by variables and constituent boundaries. Section 3 explains a method for deriving possible linglish structures. Section 4 explains structural disambiguation using distance calculations in the example-based framework. Section 5 explains an cxample of Japanese sentence analysis using our constituent botndary parsing method, and Section 6 
reports on the experimental results.

\section{Pattern}

A pattern represents maningful units for linguistic structure and transfer in TDMT, and is defined as a sequence that consists of variables and symbols representing constituent boundaries. A variable corresponds to some linguistic constituent, and a constituent boundary does not allow any two variables to be adjacent. A constituent boundary is expressed by either a functional word or a part-of-speech bigram marker ${ }^{1}$.

The explanations in this and the subsequent two sections, use English sentence parsing.

\subsection{Part-of-speech}

Table 1 shows the English parts-of-speech, currently used in our English-to-Japanese TDMT system. This part-of-specch system does not necessarily agree with that of conventional grammar.

Table 1 English parts-o[-speceh

\begin{tabular}{lll} 
part-of-specch & abbreviation & example \\
\hline adjective & adj & large \\
adverb & adv & exactly \\
interjection & interj & oh \\
common noun & noun & bus \\
numeral & num & eleven \\
proper noun & propn & Kyoto \\
pronoun & pron & I \\
wh-word & wh & what \\
verb & verb & go \\
be-verb & be & $i s$ \\
auxiliary verb & aux & cam \\
preposition & prep & at \\
conjunction & conj & but \\
determiner & det & lhe \\
sulfix & suffix & a.m. \\
\hline
\end{tabular}

In this part-of-speech system, a be-verb, auxiliary verb, preposition, conjunction, determiner, and sullix, are classified into a functional word.

\subsection{Constituent boundary marker expressed by a functional word}

One problem with pattern descriptions using surface

\footnotetext{
1 In this paper, variables, actual words, and part-ofspeech abbreviations are expressed in capital lettcrs, italics, and gothic, respectively.
}

words is the necessity for a large number of patterns. To suppress the unnecessary patterns, the surface words in patterns are in principle restricted to functional words, which occur freciuently, and which modify or relate content words ${ }^{2}$.

For instance, the expression, "go to the station" is divided into two constituents "go" and "the station", and the preposition, "to" can be identified as a constituent boundary. Therefore, in parsing "go to the station", we use the pattern, "X to Y ", which has two variables $X$ and $Y$, and a constituent boundary, "10."

\subsection{Constitucnt boundary marker expressed by a part-of-speech bigram}

The expression "I go" can be divided into two constituents " $l$ "and "go." But it has no surface word that divides the expression into two constituents. In this case, a part-of-speech bigram is used as a constituent boundary.

Suppose that a constituent $X$ is immediately followed by a constituent $Y$. We express a boundary-marker between $X$ and $Y$ by $A-B$, where $A$ is a part-of-speech abbreviation of $X$ 's last word, and $B$ is a part-of-specch abbreviation of Y's first word. For instance, "I" and "go" are a pronoun and a verb, respectively, so the marker "pron-verb" is inserted as a boundary marker into "I go". Namely, "I pron-verb go", i.c. with the boundary marker inserted into the original input, matches the pattern "X pron-verb $\mathrm{Y} . "$

\subsection{Linguistic level}

Patterns are classified into different linguistic levels to limit the explosion of structural ambiguity during parsing. Table 2 shows typical linguistic levels in English patterns.

Table 2 Typical levels in English patterns

\begin{tabular}{ll}
\multicolumn{1}{c}{ level } & example \\
\hline beginning phrase & excuse me but $\mathrm{X}$ \\
compound sentence & $\mathrm{X}$ when $\mathrm{Y}$ \\
simple sentence & I would like to $\mathrm{X}$ \\
verb phrase & $\mathrm{X}$ at $\mathrm{Y}$ \\
nom phrase & $\mathrm{X}$ of $\mathrm{Y}, \mathrm{X} a t \mathrm{Y}$ \\
compound word & $\mathrm{X}$ o'clock \\
\hline
\end{tabular}

\footnotetext{
2 Exceptions are canned expressions such as "/ would like to" and "in front of", or frequtent content words such is "what."
} 
In Table 2, beginning phrase is the highest level, and compound word is the lowest. A variable on a given level is instantiated by a string described on that same level or on a lower level. For instance, in the noun phrase " $X$ of $Y$ ", the variables, $X$ and $Y$ camot be instantiated by a simple sentence.

\section{Derivation of Possible Structures}

The algorittm for constituent boundary parsing is as follows;

(A) Assignment of morphological information to each word of an input string

(B) Insertion of constituent boundary markers

(C) Derivation of possible structures by top-down patten matching

(D) Structural disambiguation by distance calculation

Note: we will explain (A), (B) and (C) in this section, and (D) in the next section, using the following Fnglish sentence;

(1) "The bus leaves Kyoto at eleven a.m."

\subsection{Assignment of morphological information}

First, each word of the input string is assigned morphological information, such as its part-of-speceh and conjugation form. Through this assignment, we can get the following part-of-speech sequence for (I).

(2) det, noun, verb, propn, prep, num, suffix

In addition, each word is also assigned a thesaurus code for distance calculations and an index for retrieving patterns. For instance, "bus" has a thesaurus cole corresponding to the semantic attribute 'car.' Morcover', from the word " $a t$ ", we can obtain the index to the pattern "X at $Y^{\prime}$, which is found for both verb phrase and noun phrase.

\subsection{Marker insertion}

A constituent boundary marker is inserted in an impul string for pattern-matching. The marker is extracted from the part-of-speech sequence of an input sentence. Since such bigrams as det-noun belong to the same constituent, marker insertion by a part-ol-speech bigram is restricted according to the items below. (a) Neither A nor $B$ is a part-of-spech relating two constituents, such as a preposition

(b) $\mathrm{A}$ is not a part-of-speech modifying a latter constituent, such as a determiner.

(c) 13 is not a part-of-specch modifying a previous constituent, such as a suflix.

We maintain a list of part-of-speech bigrams that are eligible as markers because they satisly the above conditions. Of the bigrams in (2), "det-noun", "propnprep", "prep-num", and "num-suffix", violate the above conditions, and are of course excluded. Thus, only "noun-verb" and "verb-propn" are inserted into sentence (1), as shown in (3).

(3) "The bus noun-verb leaves verb-propn Kyoto at eleven a.m."

\subsection{Application of patterns}

Our pattern-matching method parses an input sentence in a top-down fashion. The highest level patterns of the input sentence are applied first; then pattems at lower levels are applied. The application procedure is as follows.

(I) Get indices to patterns from each word of the sentence. With these indices, pattems are retrieved and checked to determine if cach of them can match the sentence. Then execute (II).

(II) Try to apply the highest-level patterns first. If there is a pattern that can be applied, execute (IrI) with respect to the variable bindings. Otherwise, excente (IV).

(III) Try to apply surface words (content words registered in a dictionary). If the application succeds, the application lor that portion is finished sucesssfully. Ouherwise, execule (II).

(IV) If the pattern to be applied is at the lowest level, the application latis. Otherwise, lower the level of the palterns and execute (II).

If pattern application linishes successfully for all portions of an input sentence, one or more source structures are obtained: since there is a possibility that more than one pattern can be applied to an expression in step (II), structural ambiguity may occur. We seck alt possible structures by breadth-first application, and select the most plausible structure by the total distanee value (See Section 4.4). 
In step (I), indices to possible pattems are obtained from several words and bigrams in the marker-inserted sentence (3), as shown in Table 3.

Table 3 Retrieved patterns from (3)

\begin{tabular}{lll} 
word & retrieved pattern (linguistic level) \\
\hline the & the X & (compound word) \\
noun-verb & $X$ noun-verb Y (simple sentence) \\
verb-propn & $X$ verb-propn Y (verb plialse) \\
at & $X a t$ Y (verb phrase, noun phrase) \\
a.m. & $X a . m . \quad$ (compound word) \\
\hline
\end{tabular}

After step (I) is finished, steps (II)-(IV) are repeated recursively. First, the highest level pattern of the input sentence is applied. This is " $X$ noun-verb $Y$ ", which is defined at the simple sentence level. Next, an attempt is made to apply patterns to the variable bindings "the bus" and "leaves verb-propn Kyolo at eleven a.m.", which are bound to variables $X$ and $Y$, respectively. To "the bus", at compound word level patuen "the $\mathrm{X}$ " is applied first, and the surface word "bus" is applied to parse "the bus." Likewise, patterns and surlace words are applied to the remaining part, and the application is firished successfully.

The pattern " $X$ at $Y$ " is found for both verb phrase and noun phrase. "leaves verb-propn Kyoro al eleven a.m." thus has two possible structures, by the application of "X at $Y$." "X verb-propn $Y$ " at the verb phrase level and "X a.m." at compound word level, are also applied. Fig. 1 is the tree representation derived from the structure for sentence (1) where " $\mathrm{X}$ at $\mathrm{Y}$ " is a verb phrase, while Fig. 2 is a tree representation derived from the structure in which "X $a t \mathrm{Y}$ " is a noun phrase. $A$ boldface denotes the head part in each pattern. This information is utilized for extracting an input for distance calculations (Sec section 4.3).

\section{Distance Calculation}

In this section, a method for structural disambiguation utilizing distance calculation, is described.

\subsection{Distance}

The distance between two words is reduced to the distance between their respective semantic attributes in a thesaurus. Words have associated thesaurus codes, which correspond to particular semantic attributes. The distance between the semantic attributes is deternined according to the relationship of their positions in the hicrarchy of

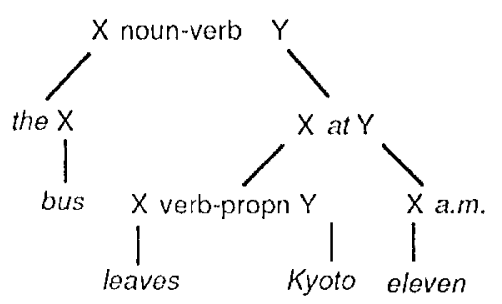

Fig. 1 Structure in which " $\mathrm{X}$ at $\mathrm{Y}$ " is a verb phrase

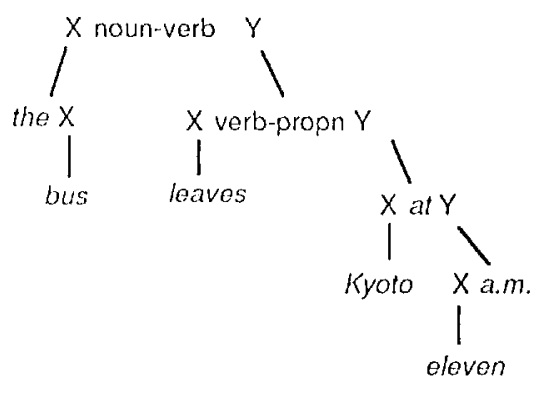

Fig. 2 Structure in which " $X$ at $Y$ " is a noun phrase

the thesaurus, and varies from 0 to 1 . The value 0 indicales that two semantic attributes belong to exactly the same category, and 1 indicates that they are unrelated.

An expression consists of words. The distance between expressions is the sum of the distance between words mulliplied by each weight.

The distance is calculated quickly because of the simple mechanism employed. (Sumita, 1992) and (Furuse, 1992, 1994) give a detailed account of the distance calculation mechanism we are adopting.

\subsection{Best-match by distance calculation}

The advantages of an example-based framework are mainly due to the distance calculation, which achieves the best-match operation between the input and provided examples.

In T'DMT, translation is performed by applying stored cmpirical transfer knowledge. In TDMT transfer knowledge, each source pattem has example words of variables and possible target patterns. The most appropriate target pattern is selected according to the calculated distance between the input words and the example words. The English pattern " $\mathrm{X}$ at $\mathrm{Y}$ " at the verb phase level, corresponds to several possible 
Japanese expressions, as shown in the following English-to-Japanese transfer knowledge:

$$
\begin{aligned}
\mathrm{X} a t \mathrm{Y} \Rightarrow \mathrm{Y}^{\prime} \text { de } \mathrm{X}^{\prime} & ((\text { prescnt, conference }) .), \\
\mathrm{Y}^{\prime} \text { ni } \mathrm{X}^{\prime} & ((\text { stay, hotel)..)}) \\
\mathrm{Y}^{\prime} \text { wo } \mathrm{X}^{\prime} & ((\text { look, it }) . .)
\end{aligned}
$$

The first possible target pattern is " $\mathrm{Y}^{\prime} d e \mathrm{X}^{\prime}$ ", with example set ((present, conference)..). We will see that this target pattern is likely to be selected to the extent that the input variable bindings are semantically similar to the example elements "present" and "conference." Within this pattern, $X$ ' is the target word corresponding to $\mathrm{X}$, the result of transfer. "present" and "conference" are sample bindings for " $\mathrm{X}$ at $\mathrm{Y}$ ", where $\mathrm{X}=$ "present", and $\mathrm{Y}=$ "conference", The above transter knowledge is compiled from such translation examples as the source-target pair of "present a paper at the conference" and "kaigi de ronbun wo happyou-suru", where "kaigi" means "conference" and "huppyou-suru" means "present".

The semantic distance from the inpul is calculated for all examples. Then the example with the least distance from the input is chosen, and the target expression of that example is extracted. If the input is closest to (stay, hotel), "Y' ni $\mathrm{X}^{\prime}$ " is chosen as the target expression.

The enrichment of examples increases the accuracy of determining the target expression and strucure because conditions become more detailed.

\subsection{Input of distance calculation}

An input for distance calculation consists of head words in variable parts. In "X at $\mathrm{Y}$ " lor the structure in Fig. $1, X$ and $Y$ are substituted for the compound expressions, "leaves verb-propn Kyoto" and "eleven a.m.", respectively. In such cases, it is necessary to extract head words as the input for the distince calculation about " $\mathrm{X} a l \mathrm{Y}$ ".

In order to get head words, the head part is designaled in each patten (boldrace in Figs. 1 and 2). For instince, the pattern " $X$ verb-propn $Y$ " contains the information that $X$ is a head part. So the head of "leaves verb-propo Kyoto" is "leaves", and the head of " $X$ a.m." is "a.m.". Thus, in "X al $\mathrm{Y}$ " for the structure in Fig. 1, the input of the distance calculation is (leaves, a.m.).

Table 4 shows the result of distance calculation in " $\mathrm{X}$ at $\mathrm{Y} "$ in Fig. 1. The most plausible target structure " $\mathrm{Y}^{\prime} n i \mathrm{X}$ " "and its distance value 0.17 are obtained by the distance calculation.

Head words are passed upward from lower patterns to higher patterns. Since the head of the verb phrase pattem, "X a $\mathrm{Y}$ " is assigned to $\mathrm{X}$, lhe head of "leaves verb-propn Kyoto at eleven a.m." is "leaves", which is the head of "leaves verb-propn Kyoto". The head of "the bus" is "bus" from the head inlormation that the

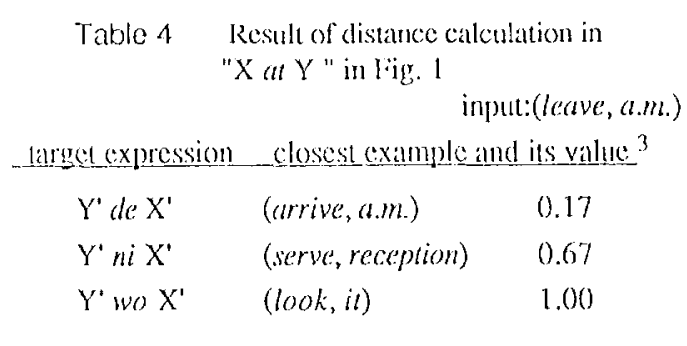

head of "the $\mathrm{X}$ " is $\mathrm{X}$. Thus, the input of the distance calculation of " $\mathrm{X}$ noun-verb $\mathrm{Y}$ " is (bus, leave).

\subsection{Structural disambiguation}

Distance catculation selects not only the most plausible target expression but also the most plausible sourec structure. When structural ambiguty occurs, the most appropriate structure is selected by computing the totals for all possible combinations of partial distance values. The structure with the least total distance is judged most consistent with empirical knowledge, and is chosen as the most plausible structure (Furuse 1992 1994; Sumita 1993).

Table 5 shows the result of each partial distance calculation for the structure in Fig. 1. From Table 5, we get the total distance value 1.17 for the structure in lïg. 1.

Table 5 Result of cach partial distance calculation for the suructure in ligg. 1

$\begin{array}{lll}\text { source } & \text { chosen target distance value } \\ \text { the } X & X^{\prime} & 0.33 \\ X \text { noun-vorb } Y & X^{\prime} \text { wa } Y^{\prime \prime} & 0.67 \\ X \text { verb-propn } Y & Y^{\prime} \text { wo } X^{\prime} & 0.00 \\ X a l Y & Y^{\prime \prime} \text { ni } X^{\prime} & 0.17 \\ X a m . & \text { gozen } X^{\prime} j i & 0.00\end{array}$

The differenee in total distance value between two possible structures for sentence (1) is due only to the distance value of "X at $\mathrm{Y}$ ", for the strucure in Figs. 1 and 2. For the structure in Fig. 2, the distance value of " $\mathrm{X}$ al $\mathrm{Y}$ " at the noun phrase level is given as 0.83 , as shown in Table 6 , and is given a total distance of 1.83 . Thus, the structure in Fig. 1 is selected as the

3 These values were computed based on the present transler knowledge of the 'IDM' system. 
appropriate result because it has the least lotal distance value.

$\begin{array}{ccc}\text { Table 6 } & \begin{array}{l}\text { Result of distance calculation in } \\ \text { "X at } \mathrm{Y}^{\prime} \text { in Fig. } 2\end{array} \\ \text { input:(Kyoto, a.m.) }\end{array}$

In machine translation, it is important to disambiguate the possible structures, because a difference in structure may bring about a translation difference. For instance, the structures in Figs, 1 and 2 give dillerent Japanese translations (4) and (5), respectively. (4) is selected because it is generated from the best structure with the least total distance value.

(4) basu wa gozen 11 ji ni Kyolo wo de masu

(5) basu wa gozen ll ji no Kyoto wo de masu

\section{Constituent Boundary Parsing in Japanese}

Since a postposition is quite often used as a caseparticle in Japanese, the boundary markers expressed by a part-of-speech bigram may not be used less frequently than in English. However, in spoken Japanese, postpositions are frequently omitted. The Japanese sentence "Kochira wa jimukyoku" where kochira means this and jimukyoku moans "office", is translated into the English sentence "This is the office" by applying transfer knowledge such as the followings:

$$
X w a Y Y^{\prime} \text { be } Y^{\prime \prime}
$$

But postpositions are often omitted in matural spoken Japanese, e.g. in the sentence "Kochira jimukyoku." The sentence can thus be divided into two noun phrases, "kochira" and "jimukyoku." "kochira" is a pronoun, and "jimukyoku" is a noun. So, using the bigram method of marking boundaries, we get "Kochira pronnoun jimukyoku", where the bigram "pron-noun" was inserted. The English sentence "This is the office" can then be produced by applying the lollowing transfer

4"basu", "de", and "masu" mean "bus", "leave", and a polite sentence-final form, respectively.

5 For simplicity, examples and other possible turget expressions are omitted. knowledge for the pattern "X pron-noun $Y$ ";

$$
X \text { pron-noun } \mathrm{Y} \Rightarrow \mathrm{X}^{\prime} \text { be } \mathrm{Y}^{\prime}
$$

In Japanese adnominal expressions, 100, constituent boundary markers are inserted between the modilier and the modilied.

\section{Results}

We have evaluated the efficiency of our parsing method by utilizing a Japanese-10-English (JE) and Englis(1-to-Japanese (EJ) TDMT protolype system (Furuse 1994; Sobashima 1994), which is running on a Symbolics XL.1200, a LISP machine with 10MIPS performance. The system's domain is incuiries concening international conference registrations. The eflicency is evaluated with 154 Japanese sentences and 138 corresponding English sentences, which are extracted from 10 dialogues in the domain. The system has about 500 source pattems for JE translation and about 350 source patterns for EJ translation.

The test sentences mentioned above have already been trained to investigate the efficiency of the method, and can be parsed correctly by the system. Table 7 outlines the 154 Japanese sentences and 138 corresponding English sentences.

Table 7 Oulline of test sentences Japanese Englishs

$\begin{array}{lll}\text { words per input sentence } & 9.8 & 8.7 \\ \text { average number of possible structures } & 1.5 & 4.8\end{array}$

An Finglish sentence tends to have more struetural ambiguities than a Japanese sentence, because of PP. altachment, the phenomenon that an English preposition produces both a noun verb phrase and a noun phase. In contrast, the Japanese postposition does not generally produce diflerent-level constituents.

Table 8 shows how much lime it takes to reach the best structure and translation output in our JE and EJ TDMT system. The processing lime for distance calculation includes structural disambiguation in addition to larget pattern selection.

This demonstrates that the our parsing method can get the best structure and transtation output quickly widhin the example-based framework. 
Table 8 Processing time for lle TI) NTl'system

\begin{tabular}{lll} 
& JE & LI. \\
\hline derivation of possible structures & $0.25(\mathrm{scc})$ & 0.17 \\
distance calculation & 1.32 & 0.14 \\
whole translation & 2.17 & 1.07 \\
\hline
\end{tabular}

\section{Concluding Remarks}

A constituent boundary parsing method lor examplebased machine translation has been proposed. l.inguistic patterns consisting of variables and constituent boundaries, are applied to an input string in al top--klown fashion, and the possible structures can be disambigutated using distance calculation by the example-based framework. This method is elficien, and uscful for parsing boh Japanese and Linglish sentences. The TDMT system, which bidirectionally translates between Japanese and English within the eximple-based framework, utilizes this parsing method and achicves efficient and robust spoken langwage translation.

By introducing linguistic information to more patterns, there is a possibility that this method can also be utilized for rule-based MT, deep semantic analysis, and so on. We will improve our parser by increasing the number of training sentences, and test its accuracy on open data.

\section{Acknowledgements}

The authors would like to thank the members of AT'R Interpreting Telecommunications Research Laboratories for their comments on various parts of this tesearch. Special thanks are due to Kohei Habara and Yasuhiro Yamazaki, for their support of this research.

\section{Bibliography}

Furnse, O., and Iida, H. (1992). Cooperation between Transfer and Analysis in Example-Based Framework. Proc. of COL.ING-92, pp.645-651.

Furuse, O., Sumita, E., and Lida, II. (1994). TransierDriven Machine Transtation Utilizing, Empirical Knowledge. Transactions of Information Processingr Society of Japan, Vol.35, No.3, pp.414.425 (in Japanesc).

Grishman, R., and Kosaka, M. (1992). Combining

\footnotetext{
6 The distance calculation tine in IJ translation is short, since the system has not yet learned enough translation examples concerning fJ translation.
}

Rationalist and Empiricist Approaches to Machine Transkatioin. Proc. of TMY-92, pp.263-274.

Jones, D. (1992). Non-hybrid Fxample-balsed Machine Translation Architectures. Proc. of TMI-92, pp.163-171.

Mclean, I. J. (1992). Example-Based Machine Transtation using Connectionist Matching. Proc. of TMI-92, pp.35-43.

Margermam, D. M., and Marcus, M. P. (1990). Parsing a Natural Language Using Mutual Information Stitistics. Proc of $A \wedge \wedge \mathrm{I} 90$, pp.984-989.

Maruyama, H., and Watanabe, H. (1992). Tree Cover Search Algorithm for Lxample-Based Transtation. Proc. of 'TMI-92, pl. 173-184.

Maruyama, H. (1993). Pallem-Based Translation: Context-Fee Transducer and Its Application to Practical N'P. Proc. of Natural Language Processing Pacilic Rim Symposium '93, pp.232-237.

Nagao, M. (1984). A ramework of a mechanical translation between Japanese and Fnglish by analogy principle. in Artificial and Human Intelligence, eds. Elithorn, A. and Bancrji, R., Nortl-Holland, pp.173180.

Nirenburg, S., Domashuev, C., and Grannes, D.J. (1993). Two Approaches to Matching in Example-Based Machine Transtation. Proce ol "I'MI-93, pp.47-57.

Sato S. (1991). Example-Based Machine Ttanslation. Doctorial 'Thesis, Kyoto University.

Sobashima, Y., Furuse, O., Akamine, S., Kawai, J., and lida, II. (1994). A Bidirectional Trmasler-Driven Machine Transtation System for Spoken Dialogues. Proc. of COL ING-94.

Sumita, Eand lida, H. (1992), Example-l3ased Transfer of Japanese Adnominal Particles into English. IleICE TRANS, INI: \& SYST, Vol.175-D, No.4, pp.58559).

Sumila, F., Furuse, O.,and lida, M. (1993). An Lxample-Based Disambiguation of Prepositional Planse Altachment. Proc. of 'I'MI-93, p[1.80-91.

Walanabe, H. (1992). Similarity-Driven Transfer System. Proc of COLING-92, pp.770-776. 